\title{
$H((\cdot, \cdot),(\cdot, \cdot))$-Mixed Cocoercive Operators with an Application for Solving Variational Inclusions in Hilbert Spaces
}

\author{
Shamshad Husain and Sanjeev Gupta \\ Department of Applied Mathematics, Faculty of Engineering and Technology, Aligarh Muslim University, Aligarh 202002, India \\ Correspondence should be addressed to Sanjeev Gupta; guptasanmp@gmail.com
}

Received 29 May 2013; Accepted 17 September 2013

Academic Editor: William Ziemer

Copyright ( 92013 S. Husain and S. Gupta. This is an open access article distributed under the Creative Commons Attribution License, which permits unrestricted use, distribution, and reproduction in any medium, provided the original work is properly cited.

\begin{abstract}
We investigate a class of new $H((\cdot, \cdot),(\cdot, \cdot))$-mixed cocoercive operators in Hilbert spaces. We extend the concept of resolvent operators associated with $H(\cdot, \cdot)$-cocoercive operators to the $H((\cdot, \cdot),(\cdot, \cdot))$-mixed cocoercive operators and prove that the resolvent operator of $H((\cdot, \cdot),(\cdot, \cdot))$-mixed cocoercive operator is single valued and Lipschitz continuous. Some examples are given to justify the definition of $H((\cdot, \cdot),(\cdot, \cdot))$-mixed cocoercive operators. Further, by using resolvent operator technique, we discuss the approximate solution and suggest an iterative algorithm for the generalized mixed variational inclusions involving $H((\cdot, \cdot),(\cdot, \cdot))$ mixed cocoercive operators in Hilbert spaces. We also discuss the convergence criteria for the iterative algorithm under some suitable conditions. Our results can be viewed as a generalization of some known results in the literature.
\end{abstract}

\section{Introduction}

In recent past, monotone mappings have a large number of applications, especially in differential equations, integral equations, mathematical economics, optimal control, and so forth. There are various kinds of generalized monotonicity such as pseudomonotone, quasimonotone, and paramonotone; see for example, [1-4]. The cocoercive mappings were studied by Tseng [5], Magnanti and Perakis [6], and Zhu and Marcotte [7] which are also the generalized forms of monotone mappings.

The resolvent operator techniques are important to study the existence of solutions and to design iterative schemes for different kinds of variational inequalities and their generalizations, which are providing mathematical models to some problems arising in optimization and control, economics, and engineering sciences. In order to study various variational inequalities and variational inclusions, Fang and Huang, Kazmi and Khan, and Lan et al. investigated many generalized operators such as $H$-monotone [8], $H$-accretive [9], $(P, \eta)$-proximal point [10], $(P, \eta)$-accretive [11], $(H, \eta)$ monotone [12], and $(A, \eta)$-accretive mappings [13]. Recently, Zou and Huang [14] introduced and studied $H(\cdot, \cdot)$-accretive operators; Kazmi et al. [15-17] introduced and studied generalized $H(\cdot, \cdot)$-accretive operators and $H(\cdot, \cdot)$ - $\eta$-proximal point mapping; $\mathrm{Xu}$ and Wang [18] introduced and studied $(H(\cdot, \cdot), \eta)$-monotone operators; Ahmad et al. [19] introduced and studied $H(\cdot, \cdot)$-cocoercive operators and Husain and Gupta $[20,21]$ introduced and studied $H(\cdot, \cdot)$-mixed operator and generalized $H(\cdot, \cdot, \cdot)-\eta$-cocoercive operators.

Motivated by the recent work going in this direction, we consider a class of $H((\cdot, \cdot),(\cdot, \cdot))$-mixed cocoercive operators, a natural generalization of monotone (accretive) operators in Hilbert (Banach) spaces. For details, we refer to see $[8,9,12-14,18-22]$. We extend the concept of resolvent operators associated with $H(\cdot, \cdot)$-cocoercive operators to the $H((\cdot, \cdot),(\cdot, \cdot))$-mixed cocoercive operators and prove that the resolvent operator of $H((\cdot, \cdot),(\cdot, \cdot))$-mixed cocoercive operator is single valued and Lipschitz continuous. Further, we consider the generalized mixed variational inclusion problem involving $H((\cdot, \cdot),(\cdot, \cdot))$-mixed cocoercive operator in Hilbert spaces. Using new resolvent operator technique, we prove the existence of solutions and suggest an iterative algorithm for the generalized mixed variational inclusions. Furthermore, we discuss the convergence criteria of the iterative algorithm under some suitable conditions. Our results can be viewed as 
an extension and generalization of some known results [1422]. For illustration of Definitions 4 and 7 and Theorem 20, Examples 5, 8, and 21 are given, respectively.

\section{Preliminaries}

Let $X$ be a real Hilbert space endowed with a norm $\|\cdot\|$ and an inner product $\langle\cdot, \cdot\rangle, d$ the metric induced by the norm $\|\cdot\|, 2^{X}$ (resp., $C B(X)$ ) the family of all nonempty (resp., closed and bounded) subsets of $X$, and $\mathscr{D}(\cdot, \cdot)$ the Hausdorff metric on $C B(X)$ defined by

$$
\mathscr{D}(P, Q)=\max \left\{\sup _{x \in P} d(x, Q), \sup _{y \in Q} d(P, y)\right\},
$$

where $d(x, Q)=\inf _{y \in \mathrm{Q}}\|x-y\|$ and $d(P, y)=\inf _{x \in P}\|x-y\|$.

In the sequel, we recall important basic concepts and definitions, which will be used in this work.

Definition 1. A mapping $g: X \rightarrow X$ is said to be

(i) monotone if

$$
\langle g(x)-g(y), x-y\rangle \geq 0, \quad \forall x, y \in X
$$

(ii) $\xi$-strongly monotone if there exists a constant $\xi>0$ such that

$$
\langle g(x)-g(y), x-y\rangle \geq \xi\|x-y\|^{2}, \quad \forall x, y \in X
$$

(iii) $\mu$-cocoercive if there exists a constant $\mu>0$ such that

$$
\langle g(x)-g(y), x-y\rangle \geq \mu\|g(x)-g(y)\|^{2}, \quad \forall x, y \in X ;
$$

(iv) $\gamma$-relaxed cocoercive if there exists a constant $\gamma>0$ such that

$$
\langle g(x)-g(y), x-y\rangle \geq-\gamma\|g(x)-g(y)\|^{2}, \quad \forall x, y \in X
$$

(v) $\zeta_{g}$-Lipschitz continuous if there exists a constant $\zeta_{g}>$ 0 such that

$$
\|g(x)-g(y)\| \leq \zeta_{g}\|x-y\|, \quad \forall x, y \in X
$$

(vi) $\alpha$-expansive if there exists a constant $\alpha>0$ such that

$$
\|g(x)-g(y)\| \geq \alpha\|x-y\|, \quad \forall x, y \in X
$$

if $\alpha=1$, then it is expansive.

Definition 2. A set-valued mapping $M: X \rightarrow 2^{X}$ is said to be $m$-relaxed monotone if there exists a constant $m>0$ such that

$$
\begin{array}{r}
\langle u-v, x-y\rangle \geq-m\|x-y\|^{2}, \quad \forall x, y \in X, \\
u \in M(x), \quad v \in M(y) .
\end{array}
$$

Definition 3 (see $[14,19]$ ). Let $H: X \times X \rightarrow X$ and $A, B$ : $X \rightarrow X$ be the single-valued mappings. Then

(i) $H(A, \cdot)$ is said to be $\alpha$-strongly monotone with respect to $A$ if there exists a constant $\alpha>0$ such that

$$
\begin{array}{r}
\langle H(A x, u)-H(A y, u), x-y\rangle \geq \alpha\|x-y\|^{2}, \\
\forall x, y, u \in X ;
\end{array}
$$

(ii) $H(\cdot, B)$ is said to be $\beta$-relaxed monotone with respect to $B$ if there exists a constant $\beta>0$ such that

$$
\begin{array}{r}
\langle H(u, B x)-H(u, B y), x-y\rangle \geq-\beta\|x-y\|^{2}, \\
\forall x, y, u \in X ;
\end{array}
$$

(iii) $H(A, \cdot)$ is said to be $\mu$-cocoercive with respect to $A$ if there exists a constant $\mu>0$ such that

$$
\begin{array}{r}
\langle H(A x, u)-H(A y, u), x-y\rangle \geq \mu\|A x-A y\|^{2}, \\
\forall x, y, u \in X ;
\end{array}
$$

(iv) $H(\cdot, B)$ is said to be $\gamma$-relaxed cocoercive with respect to $B$ if there exists a constant $\gamma>0$ such that

$$
\begin{array}{r}
\langle H(u, B x)-H(u, B y), x-y\rangle \geq-\gamma\|B x-B y\|^{2}, \\
\forall x, y, u \in X ;
\end{array}
$$

(v) $H(A, \cdot)$ is said to be $\tau_{1}$-Lipschitz continuous with respect to $A$ if there exists a constant $\tau_{1}>0$ such that

$$
\|H(A x, \cdot)-H(A y, \cdot)\| \leq \tau_{1}\|x-y\|, \quad \forall x, y \in X,
$$

(vi) $H(\cdot, B)$ is said to be $\tau_{2}$-Lipschitz continuous with respect to $B$ if there exists a constant $\tau_{2}>0$ such that

$$
\|H(\cdot, B x)-H(\cdot, B y)\| \leq \tau_{2}\|x-y\|, \quad \forall x, y \in X .
$$

Definition 4. Let $H:(X \times X) \times(X \times X) \rightarrow X$ and $A, B, C, D$ : $X \rightarrow X$ be the single-valued mappings. Then

(i) $H((A, \cdot),(C, \cdot))$ is said to be $\left(\mu_{1}, \gamma_{1}\right)$-mixed cocoercive with respect to $(A, C)$ if there exist constants $\mu_{1}, \gamma_{1}>0$ such that

$$
\begin{array}{r}
\langle H((A x, u),(C x, u))-H((A y, u),(C y, u)), x-y\rangle \\
\geq \mu_{1}\|A x-A y\|^{2}+\gamma_{1}\|x-y\|^{2}, \quad \forall x, y, u \in X
\end{array}
$$

(ii) $H((\cdot, B),(\cdot, D))$ is said to be $\left(\mu_{2}, \gamma_{2}\right)$-relaxed mixed cocoercive with respect to $(B, D)$ if there exist constants $\mu_{2}, \gamma_{2}>0$ such that

$$
\begin{array}{r}
\langle H((u, B x),(u, D x))-H((u, B y),(u, D y)), x-y\rangle \\
\quad \geq-\mu_{2}\|B x-B y\|^{2}+\gamma_{2}\|x-y\|^{2}, \quad \forall x, y, u \in X ;
\end{array}
$$


(iii) $H((A, B),(C, D))$ is said to be $\tau$-mixed Lipschitz continuous with respect to $A, B, C$, and $D$ if there exists a constant $\tau>0$ such that

$$
\begin{aligned}
& \|H((A x, B x),(C x, D x))-H((A y, B y),(C y, D y))\| \\
& \quad \leq \tau\|x-y\|, \quad \forall x, y \in X .
\end{aligned}
$$

Example 5. Let $X=\mathbb{R}^{2}$ with usual inner product. Let $A, B, C, D: \mathbb{R}^{2} \rightarrow \mathbb{R}^{2}$ be defined by

$$
\begin{aligned}
A x=\left(\begin{array}{l}
4 x_{1} \\
4 x_{2}
\end{array}\right), \quad B y=\left(\begin{array}{l}
-3 y_{1} \\
-3 y_{2}
\end{array}\right), \\
C x=\left(\begin{array}{l}
2 x_{1} \\
2 x_{2}
\end{array}\right), \quad D y=\left(\begin{array}{l}
y_{1} \\
y_{2}
\end{array}\right), \\
\\
\quad \forall\left(x_{1}, x_{2}\right),\left(y_{1}, y_{2}\right) \in \mathbb{R}^{2} .
\end{aligned}
$$

Suppose that $H:\left(\mathbb{R}^{2} \times \mathbb{R}^{2}\right) \times\left(\mathbb{R}^{2} \times \mathbb{R}^{2}\right) \rightarrow \mathbb{R}^{2}$ is defined by

$$
H((A x, B y),(C x, D y))=A x+B y+C x+D y .
$$

Then $H((A, B),(C, D))$ is $(1 / 4,2)$-mixed cocoercive with respect to $(A, C),(1 / 3,1)$-relaxed mixed cocoercive with respect to $(B, D)$, and 4-mixed Lipschitz continuous with respect to $A, B, C$, and $D$.

Indeed, let, for any $u \in X$,

$$
\begin{aligned}
\langle H & ((A x, u),(C x, u)) \\
& -H((A y, u),(C y, u)), x-y\rangle \\
= & \langle A x+C x-A y-C y, x-y\rangle \\
= & \langle A x-A y, x-y\rangle+\langle C x-C y, x-y\rangle \\
= & \left\langle\left(4 x_{1}-4 y_{1}, 4 x_{2}-4 y_{2}\right),\right. \\
& \left.\left(x_{1}-y_{1}, x_{2}-y_{2}\right)\right\rangle \\
& +\left\langle\left(2 x_{1}-2 y_{1}, 2 x_{2}-2 y_{2}\right),\right. \\
& \left.\left(x_{1}-y_{1}, x_{2}-y_{2}\right)\right\rangle \\
= & 4\left(x_{1}-y_{1}\right)^{2}+4\left(x_{2}-y_{2}\right)^{2}+2\left(x_{1}-y_{1}\right)^{2} \\
& +2\left(x_{2}-y_{2}\right)^{2} \\
= & 4\|x-y\|^{2}+2\|x-y\|^{2}, \\
\|A x-A y\|^{2}= & \langle A x-A y, A x-A y\rangle \\
=\langle & \left\langle\left(4 x_{1}-4 x_{2}, 4 y_{1}-4 y_{2}\right),\right. \\
& \left.\left(4 x_{1}-4 x_{2}, 4 y_{1}-4 y_{2}\right)\right\rangle
\end{aligned}
$$

$$
\begin{aligned}
& =16\left(x_{1}-y_{1}\right)^{2}+16\left(x_{2}-y_{2}\right)^{2}, \\
& \langle H((A x, u),(C x, u)) \\
& \quad-H((A y, u),(C y, u)), x-y\rangle \\
& \quad \geq \frac{1}{4}\|A x-A y\|^{2}+2\|x-y\|^{2},
\end{aligned}
$$

that is, $H((A, B),(C, D))$ is $(1 / 4,2)$-mixed cocoercive with respect to $(A, C)$. Consider

$$
\begin{aligned}
\langle H & ((u, B x),(u, D x)) \\
& -H((u, B y),(u, D y)), x-y\rangle \\
= & \langle B x+D x-B y-D y, x-y\rangle \\
= & \langle B x-B y, x-y\rangle+\langle D x-D y, x-y\rangle \\
= & \left\langle\left(-3 x_{1}+3 y_{1},-3 x_{2}+3 y_{2}\right),\right. \\
& \left.\left(x_{1}-y_{1}, x_{2}-y_{2}\right)\right\rangle \\
& +\left\langle\left(x_{1}-y_{1}, x_{2}-y_{2}\right),\left(x_{1}-y_{1}, x_{2}-y_{2}\right)\right\rangle \\
= & -3\left(x_{1}-y_{1}\right)^{2}-3\left(x_{2}-y_{2}\right)^{2}+\left(x_{1}-y_{1}\right)^{2} \\
& +\left(x_{2}-y_{2}\right)^{2} \\
= & -3\|x-y\|^{2}+\|x-y\|^{2}, \\
\|B x-B y\|^{2}=\langle & B x-B y, B x-B y\rangle \\
= & \left\langle\left(-3 x_{1}+3 y_{1},-3 x_{2}+3 y_{2}\right),\right. \\
& \left.\left(-3 x_{1}+3 y_{1},-3 x_{2}+3 y_{2}\right)\right\rangle \\
=-9 & \left\{\left(x_{1}-y_{1}\right)^{2}+\left(x_{2}-y_{2}\right)^{2}\right\}, \\
\langle H & ((u, B x),(u, D x)) \\
& -H((u, B y),(u, D y)), x-y\rangle \\
\geq & -\frac{1}{3}\|B x-B y\|^{2}+\|x-y\|^{2} ; \\
&
\end{aligned}
$$

that is, $H((A, B),(C, D))$ is $(1 / 3,1)$-relaxed mixed cocoercive with respect to $(B, D)$.

$$
\begin{gathered}
\|H((A x, B x),(C x, D x))-H((A y, B y),(C y, D y))\|^{2} \\
=\langle H((A x, B x),(C x, D x))-H((A y, B y),(C y, D y)), \\
H((A x, B x),(C x, D x))-H((A y, B y),(C y, D y))\rangle
\end{gathered}
$$




$$
\begin{aligned}
&=\langle(A x+B x+C x+D x)-(A y+B y+C y+D y), \\
&(A x+B x+C x+D x)-(A y+B y+C y+D y)\rangle \\
&=\left\langle\left(4 x_{1}, 4 x_{2}\right)-\left(4 y_{1}, 4 y_{2}\right),\left(4 x_{1}, 4 x_{2}\right)-\left(4 y_{1}, 4 y_{2}\right)\right\rangle \\
&= 16\left\{\left(x_{1}-y_{1}\right)^{2}+\left(x_{2}-y_{2}\right)^{2}\right\}, \\
&\|H((A x, B x),(C x, D x))-H((A y, B y),(C y, D y))\| \\
& \leq 4\|x-y\| ;
\end{aligned}
$$

that is, $H((A, B),(C, D))$ is 4-mixed Lipschitz continuous with respect to $A, B, C$, and $D$.

Definition 6. Let $H:(X \times X) \times(X \times X) \rightarrow X, P: X \times X \rightarrow X$, and $A, B, C, D: X \rightarrow X$ be the single valued mappings and $S, T: X \rightarrow C B(X)$ the set-valued mappings. The mapping $H((A, B),(C, D))$ is said to be $v$-mixed strongly monotone with respect to $P, S$, and $T$ if and only if there exists a constant $v>0$ such that

$$
\begin{aligned}
& \left\langle H\left(\left(A u_{n}, B u_{n}\right),\left(C u_{n}, D u_{n}\right)\right)\right. \\
& -H\left(\left(A u_{n-1}, B u_{n-1}\right),\left(C u_{n-1}, D u_{n-1}\right)\right), \\
& \left.P\left(x_{n}, y_{n}\right)-P\left(x_{n-1}, y_{n-1}\right)\right\rangle \leq v\left\|u_{n}-u_{n-1}\right\|^{2}, \\
& \forall u_{n}, u_{n-1} \in X, x_{n} \in S\left(u_{n}\right), y_{n} \in T\left(u_{n}\right), \\
& x_{n-1} \in S\left(u_{n-1}\right), y_{n-1} \in T\left(u_{n-1}\right) .
\end{aligned}
$$

\section{3. $H((\cdot, \cdot),(\cdot, \cdot))$-Mixed Cocoercive Operators}

This section deals with a new concept and properties of $H((\cdot, \cdot),(\cdot, \cdot))$-mixed cocoercive mappings, which provides a unifying framework for the existing cocoercive operators, monotone operators in Hilbert spaces, and accretive operators in Banach space.

Definition 7. Let $H:(X \times X) \times(X \times X) \rightarrow X$ and $A, B, C, D: X \rightarrow X$ be five single-valued mappings. Let $H((A, B),(C, D))$ be $\left(\mu_{1}, \gamma_{1}\right)$-mixed cocoercive with respect to $(A, C)$ and $\left(\mu_{2}, \gamma_{2}\right)$-relaxed mixed cocoercive with respect to $(B, D)$. Then the set-valued mapping $M: X \rightarrow 2^{X}$ is said to be $H((\cdot, \cdot),(\cdot, \cdot))$-mixed cocoercive with respect to $(A, C)$ and $(B, D)$ (or simply $H((\cdot, \cdot),(\cdot, \cdot))$-mixed cocoercive in the sequel) if

(i) $M$ is $m$-relaxed monotone;

(ii) $(H((\cdot, \cdot),(\cdot, \cdot))+\rho M)(X)=X$, for all $\rho>0$.

Example 8. Let $X, H, A, B, C$, and $D$ be the same as in Example 5, and let $M: \mathbb{R}^{2} \rightarrow \mathbb{R}^{2}$ be defined by $M(x)=$ $\left(-2 \pi,-2 x_{2}\right), \forall x=\left(x_{1}, x_{2}\right) \in \mathbb{R}^{2}$.
We claim that $M$ is 2-relaxed monotone mapping. Indeed, for any $x=\left(x_{1}, x_{2}\right), y=\left(y_{1}, y_{2}\right) \in \mathbb{R}^{2}$, we have

$$
\begin{aligned}
\langle M & x-M y, x-y\rangle \\
& =\left\langle\left(-2 \pi,-2 x_{2}\right)-\left(-2 \pi,-2 y_{2}\right),\left(x_{1}-y_{1}, x_{2}-y_{2}\right)\right\rangle \\
& =\left\langle\left(0,-2\left(x_{2}-y_{2}\right)\right),\left(x_{1}-y_{1}, x_{2}-y_{2}\right)\right\rangle \\
& =-2\left(x_{2}-y_{2}\right)^{2} \\
& \geq-2\left\{\left(x_{1}-y_{1}\right)^{2}+\left(x_{2}-y_{2}\right)^{2}\right\} \\
& =-2\|x-y\|^{2} \\
\langle M & x-M y, x-y\rangle \geq-2\|x-y\|^{2} .
\end{aligned}
$$

Furthermore, $M$ is also an $H((\cdot, \cdot),(\cdot, \cdot))$-mixed cocoercive operator since $(H((\cdot, \cdot),(\cdot, \cdot))+\rho M)\left(\mathbb{R}^{2}\right)=\mathbb{R}^{2}$ for any $\rho>0$.

Remark 9.

(i) If $H((A, B),(C, D))=H(A, B)$ and $M$ is cocoercive, then $H((\cdot, \cdot),(\cdot, \cdot))$-mixed cocoercive operator reduces to $H(\cdot, \cdot)$-cocoercive operator which was studied in [19].

(ii) If $H((A, B),(C, D))=H(A, B)$ and $M$ is $m-\eta$-relaxed monotone, then $H((\cdot, \cdot),(\cdot, \cdot))$-mixed cocoercive operator reduces to $(H(\cdot, \cdot), \eta)$-monotone operator which was studied in [18].

(iii) If $H((A, B),(C, D))=H(A, B)$ and $M$ is accretive, then $H((\cdot, \cdot),(\cdot, \cdot))$-mixed cocoercive operator reduces to $H(\cdot, \cdot)$-accretive operator which was studied in [14].

(iv) If $H((A, B),(C, D))=H$, then $H((\cdot, \cdot),(\cdot, \cdot))$-mixed cocoercive operator reduces to $H$-monotone operator which was studied in $[8,9]$.

Since $H((\cdot, \cdot),(\cdot, \cdot))$-mixed cocoercive operator is a generalization of the maximal monotone operator, it is sensible that there are similar properties between them. The following result confirms this expectation.

Proposition 10. Let set-valued mapping $M: X \rightarrow 2^{X}$ be a $H((\cdot, \cdot),(\cdot, \cdot))$-mixed cocoercive operator with respect to $(A, C)$ and $(B, D)$. If $A$ is $\alpha$-expansive, $B$ is $\beta$-Lipschitz continuous, and $\mu_{1}>\mu_{2}, \alpha>\beta, \gamma_{1}, \gamma_{2}>0$ with $r=\left(\mu_{1} \alpha^{2}-\mu_{2} \beta^{2}\right)+\left(\gamma_{1}+\right.$ $\left.\gamma_{2}\right)>m$, then the following inequality

$$
\langle u-v, x-y\rangle \geq 0,
$$

holds for all $(y, v) \in \operatorname{Graph}(M)$, implies $u \in M x$, where

$$
\operatorname{Graph}(M)=\{(a, b) \in X \times X: b \in M(a)\} .
$$

Proof. Suppose on contrary that there exists $\left(x_{0}, u_{0}\right) \notin$ $\operatorname{Graph}(M)$ such that

$$
\left\langle u_{0}-v, x_{0}-y\right\rangle \geq 0, \quad \forall(y, v) \in \operatorname{Graph}(M) .
$$


Since $M$ is $H((\cdot, \cdot),(\cdot, \cdot))$-mixed cocoercive, we know $(H((\cdot, \cdot),(\cdot, \cdot))+\rho M)(X)=X$ that holds for all $\rho>0$, and so there exists $\left(x_{1}, u_{1}\right) \in \operatorname{Graph}(M)$ such that

$$
\begin{aligned}
& H\left(\left(A x_{0}, B x_{0}\right),\left(C x_{0}, D x_{0}\right)\right)+\rho u_{0} \\
& \quad=H\left(\left(A x_{1}, B x_{1}\right),\left(C x_{1}, D x_{1}\right)\right)+\rho u_{1} \in X
\end{aligned}
$$

Now,

$$
\begin{aligned}
\rho u_{0}-\rho u_{1}= & H\left(\left(A x_{1}, B x_{1}\right),\left(C x_{1}, D x_{1}\right)\right) \\
- & H\left(\left(A x_{0}, B x_{0}\right),\left(C x_{0}, D x_{0}\right)\right) \in X . \\
\left\langle\rho u_{0}-\rho u_{1}, x_{0}-x_{1}\right\rangle & =-\left\langle H\left(\left(A x_{0}, B x_{0}\right),\left(C x_{0}, D x_{0}\right)\right)\right. \\
& \left.-H\left(\left(A x_{1}, B x_{1}\right),\left(C x_{1}, D x_{1}\right)\right), x_{0}-x_{1}\right\rangle .
\end{aligned}
$$

Setting $(y, v)=\left(x_{1}, u_{1}\right)$ in (27) and then from the resultant, (28) and $m$-relaxed monotonicity of $M$, we obtain

$$
\begin{aligned}
-m\left\|x_{0}-x_{1}\right\|^{2} \leq & \rho\left\langle u_{0}-u_{1}, x_{0}-x_{1}\right\rangle \\
= & -\left\langle H\left(\left(A x_{0}, B x_{0}\right),\left(C x_{0}, D x_{0}\right)\right)\right. \\
& \left.-H\left(\left(A x_{1}, B x_{1}\right),\left(C x_{1}, D x_{1}\right)\right), x_{0}-x_{1}\right\rangle \\
= & -\left\langle H\left(\left(A x_{0}, B x_{0}\right),\left(C x_{0}, D x_{0}\right)\right)\right. \\
& \left.-H\left(\left(A x_{1}, B x_{0}\right),\left(C x_{1}, D x_{0}\right)\right), x_{0}-x_{1}\right\rangle \\
- & \left\langle H\left(\left(A x_{1}, B x_{0}\right),\left(C x_{1}, D x_{0}\right)\right)\right. \\
& \left.-H\left(\left(A x_{1}, B x_{1}\right),\left(C x_{1}, D x_{1}\right)\right), x_{0}-x_{1}\right\rangle .
\end{aligned}
$$

Since $H((A, B),(C, D))$ is $\left(\mu_{1}, \gamma_{1}\right)$-mixed cocoercive with respect to $(A, C)$ and $\left(\mu_{2}, \gamma_{2}\right)$-relaxed mixed cocoercive with respect to $(B, D)$ and $A$ is $\alpha$-expansive and $B$ is $\beta$-Lipschitz continuous; thus, (30) becomes

$$
\begin{aligned}
-m\left\|x_{0}-x_{1}\right\|^{2} \leq & -\mu_{1}\left\|A x_{0}-A x_{1}\right\|^{2}-\gamma_{1}\left\|x_{0}-x_{1}\right\|^{2} \\
& +\mu_{2}\left\|B x_{0}-B x_{1}\right\|^{2}-\gamma_{2}\left\|x_{0}-x_{1}\right\|^{2} \\
\leq & -\mu_{1} \alpha^{2}\left\|x_{0}-x_{1}\right\|^{2}-\gamma_{1}\left\|x_{0}-x_{1}\right\|^{2} \\
& +\mu_{2} \beta^{2}\left\|x_{0}-x_{1}\right\|^{2}-\gamma_{2}\left\|x_{0}-x_{1}\right\|^{2} \\
= & -\left[\left(\mu_{1} \alpha^{2}-\mu_{2} \beta^{2}\right)+\left(\gamma_{1}+\gamma_{2}\right)\right]\left\|x_{0}-x_{1}\right\|^{2} \\
= & -r\left\|x_{0}-x_{1}\right\|^{2}, \text { where } \\
r= & \left(\mu_{1} \alpha^{2}-\mu_{2} \beta^{2}\right)+\left(\gamma_{1}+\gamma_{2}\right) \\
\leq & -(r-m)\left\|x_{0}-x_{1}\right\|^{2} \leq 0,
\end{aligned}
$$

which gives $x_{0}=x_{1}$ since $r>m$. By (27), we have $u_{0}=u_{1}$, a contradiction. This completes the proof.
Theorem 11. Let the set-valued mapping $M: X \rightarrow 2^{X}$ be a $H((\cdot, \cdot),(\cdot, \cdot))$-mixed cocoercive operator with respect to $(A, C)$ and $(B, D)$. If $A$ is $\alpha$-expansive, $B$ is $\beta$-Lipschitz continuous, and $\mu_{1}>\mu_{2}, \alpha>\beta, \gamma_{1}, \gamma_{2}>0$ with $r=\left(\mu_{1} \alpha^{2}-\mu_{2} \beta^{2}\right)+\left(\gamma_{1}+\right.$ $\left.\gamma_{2}\right)>\rho m$, then $(H((A, B),(C, D))+\rho M)^{-1}$ is single valued.

Proof. For any given $x \in X$, let $u, v \in(H((A, B),(C, D))+$ $\rho M)^{-1}(x)$. It follows that

$$
\begin{gathered}
-H((A u, B u),(C u, D u))+x \in \rho M u, \\
-H((A v, B v),(C v, D v))+x \in \rho M v .
\end{gathered}
$$

Since $M$ is $m$-relaxed monotone, we have

$$
\begin{gathered}
-m\|u-v\|^{2} \\
\leq \frac{1}{\rho}\langle-H((A u, B u),(C u, D u)) \\
\quad+x-(-H((A v, B v),(C v, D v))+x), u-v\rangle \\
-\rho m\|u-v\|^{2} \\
\leq\langle-H((A u, B u),(C u, D u)) \\
+x-(-H((A v, B v),(C v, D v))+x), u-v\rangle \\
=-\langle H((A u, B u),(C u, D u)) \\
\quad-H((A v, B v),(C v, D v)), u-v\rangle \\
=-\langle H((A u, B u),(C u, D u)) \\
\quad-H((A v, B u),(C v, D u)), u-v\rangle \\
-\langle H((A v, B u),(C v, D u)) \\
-H((A v, B v),(C v, D v)), u-v\rangle .
\end{gathered}
$$

Since $H((A, B),(C, D))$ is $\left(\mu_{1}, \gamma_{1}\right)$-mixed cocoercive with respect to $(A, C)$ and $\left(\mu_{2}, \gamma_{2}\right)$-relaxed mixed cocoercive with respect to $(B, D)$ and $A$ is $\alpha$-expansive and $B$ is $\beta$-Lipschitz continuous; thus, (33) becomes

$$
\begin{aligned}
-\rho m\|u-v\|^{2} \leq & -\mu_{1}\|A u-A v\|^{2}-\gamma_{1}\|u-v\|^{2} \\
& +\mu_{2}\|B u-B v\|^{2}-\gamma_{2}\|u-v\|^{2} \\
\leq & -\mu_{1} \alpha^{2}\|u-v\|^{2}-\gamma_{1}\|u-v\|^{2} \\
& +\mu_{2} \beta^{2}\|u-v\|^{2}-\gamma_{2}\|u-v\|^{2} \\
= & -\left[\left(\mu_{1} \alpha^{2}-\mu_{2} \beta^{2}\right)+\left(\gamma_{1}+\gamma_{2}\right)\right]\|u-v\|^{2} \\
= & -r\|u-v\|^{2}, \text { where } \\
r= & \left(\mu_{1} \alpha^{2}-\mu_{2} \beta^{2}\right)+\left(\gamma_{1}+\gamma_{2}\right) \\
= & -(r-\rho m)\|u-v\|^{2} \leq 0,
\end{aligned}
$$

since $r>\rho m$. Hence, it follows that $\|u-v\| \leq 0$. This implies that $u=v$, and so $(H((A, B),(C, D))+\rho M)^{-1}$ is single valued. 
Definition 12. Let set-valued mapping $M: X \rightarrow 2^{X}$ be a $H((\cdot, \cdot),(\cdot, \cdot))$-mixed cocoercive operator with respect to $(A, C)$ and $(B, D)$. If $A$ is $\alpha$-expansive, $B$ is $\beta$-Lipschitz continuous, and $\mu_{1}>\mu_{2}, \alpha>\beta, \gamma_{1}, \gamma_{2}>0$ with $r=$ $\left(\mu_{1} \alpha^{2}-\mu_{2} \beta^{2}\right)+\left(\gamma_{1}+\gamma_{2}\right)>\rho m$, then the resolvent operator $R_{\rho, M}^{H((\cdot, \cdot)(\cdot, \cdot))}: X \rightarrow X$ is defined by

$$
R_{\rho, M}^{H((\cdot \cdot))(\cdot \cdot))}(u)=(H((A, B),(C, D))+\rho M)^{-1}(u),
$$

$\forall u \in X$

Now we prove that the resolvent operator defined by (35) is Lipschitz continuous.

Theorem 13. Let set-valued mapping $M: X \rightarrow 2^{X}$ be a $H((\cdot, \cdot),(\cdot, \cdot))$-mixed cocoercive operator with respect to $(A, C)$ and $(B, D)$. If $A$ is $\alpha$-expansive, $B$ is $\beta$-Lipschitz continuous, and $\mu_{1}>\mu_{2}, \alpha>\beta, \gamma_{1}, \gamma_{2}>0$ with $r=\left(\mu_{1} \alpha^{2}-\mu_{2} \beta^{2}\right)+\left(\gamma_{1}+\right.$ $\left.\gamma_{2}\right)>\rho m$, then the resolvent operator $R_{\rho, M}^{H((\cdot, \cdot)(\cdot, \cdot))}: X \rightarrow X$ is $1 /(r-\rho m)$-Lipschitz continuous; that is,

$$
\begin{aligned}
& \left\|R_{\rho, M}^{H((\cdot),(\cdot, \cdot))}(u)-R_{\rho, M}^{H((\cdot,),(\cdot,))}(v)\right\| \\
& \quad \leq \frac{1}{r-\rho m}\|u-v\|, \quad \forall u, v \in X .
\end{aligned}
$$

Proof. Let $u, v \in X$ be any given points. It follows from (35) that

$$
\begin{aligned}
& R_{\rho, M}^{H((\cdot,))(\cdot, \cdot))}(u)=(H((A, B),(C, D))+\rho M)^{-1}(u), \\
& R_{\rho, M}^{H((\cdot,),(\cdot, \cdot))}(v)=(H((A, B),(C, D))+\rho M)^{-1}(v), \\
& \frac{1}{\rho}\left(u-H\left(\left(A\left(R_{\rho, M}^{H((\cdot \cdot)(\cdot, \cdot))}(u)\right), B\left(R_{\rho, M}^{H((\cdot))(\cdot, \cdot))}(u)\right)\right),\right.\right. \\
& \left.\left.\left(C\left(R_{\rho, M}^{H((\cdot,))(\cdot \cdot \cdot))}(u)\right), D\left(R_{\rho, M}^{H((\cdot,)(\cdot, \cdot))}(u)\right)\right)\right)\right) \\
& \in M\left(R_{\rho, M}^{H((\cdot), \cdot(\cdot \cdot))}(u)\right), \\
& \frac{1}{\rho}\left(v-H\left(\left(A\left(R_{\rho, M}^{H((\cdot,)(\cdot, \cdot))}(v)\right), B\left(R_{\rho, M}^{H((\cdot,)(\cdot, \cdot))}(v)\right)\right),\right.\right. \\
& \left.\left.\left(C\left(R_{\rho, M}^{H((\cdot,),(\cdot, \cdot))}(v)\right), D\left(R_{\rho, M}^{H((\cdot,)(\cdot, \cdot))}(v)\right)\right)\right)\right) \\
& \in M\left(R_{\rho, M}^{H((\cdot \cdot),(\cdot \cdot))}(v)\right) \text {. }
\end{aligned}
$$

Let $z_{1}=R_{\rho, M}^{H((\cdot,)(\cdot, \cdot))}(u)$ and $z_{2}=R_{\rho, M}^{H((\cdot,)(\cdot, \cdot))}(v)$. Since $M$ is $m$ relaxed monotone, we have

$$
\begin{aligned}
\left\langle\frac { 1 } { \rho } \left( u-H\left(\left(A\left(z_{1}\right), B\left(z_{1}\right)\right),\left(C\left(z_{1}\right), D\left(z_{1}\right)\right)\right)\right.\right. \\
\left.\quad-\left(v-H\left(\left(A\left(z_{2}\right), B\left(z_{2}\right)\right),\left(C\left(z_{2}\right), D\left(z_{2}\right)\right)\right)\right)\right), \\
\left.z_{1}-z_{2}\right\rangle \geq-m\left\|z_{1}-z_{2}\right\|^{2},
\end{aligned}
$$

$$
\begin{gathered}
\left\langle\frac { 1 } { \rho } \left( u-v-H\left(\left(A\left(z_{1}\right), B\left(z_{1}\right)\right),\left(C\left(z_{1}\right), D\left(z_{1}\right)\right)\right)\right.\right. \\
\left.+H\left(\left(A\left(z_{2}\right), B\left(z_{2}\right)\right),\left(C\left(z_{2}\right), D\left(z_{2}\right)\right)\right)\right) \\
\left.z_{1}-z_{2}\right\rangle \geq-m\left\|z_{1}-z_{2}\right\|^{2}
\end{gathered}
$$

which implies that

$$
\begin{aligned}
& \left\langle u-v, z_{1}-z_{2}\right\rangle \\
& \geq\left\langle H\left(\left(A\left(z_{1}\right), B\left(z_{1}\right)\right),\left(C\left(z_{1}\right), D\left(z_{1}\right)\right)\right)\right. \\
& \left.\quad-H\left(\left(A\left(z_{2}\right), B\left(z_{2}\right)\right),\left(C\left(z_{2}\right), D\left(z_{2}\right)\right)\right), z_{1}-z_{2}\right\rangle \\
& \quad-\rho m\left\|z_{1}-z_{2}\right\|^{2} .
\end{aligned}
$$

Further, we have

$$
\begin{aligned}
& \|u-v\|\left\|z_{1}-z_{2}\right\| \\
& \geq\left\langle u-v, z_{1}-z_{2}\right\rangle \\
& \geq\left\langle H\left(\left(A\left(z_{1}\right), B\left(z_{1}\right)\right),\left(C\left(z_{1}\right), D\left(z_{1}\right)\right)\right)\right. \\
& \left.-H\left(\left(A\left(z_{2}\right), B\left(z_{2}\right)\right),\left(C\left(z_{2}\right), D\left(z_{2}\right)\right)\right), z_{1}-z_{2}\right\rangle \\
& \text { - } \rho m\left\|z_{1}-z_{2}\right\|^{2} \\
& =\left\langle H\left(\left(A\left(z_{1}\right), B\left(z_{1}\right)\right),\left(C\left(z_{1}\right), D\left(z_{1}\right)\right)\right)\right. \\
& \left.-H\left(\left(A\left(z_{2}\right), B\left(z_{1}\right)\right),\left(C\left(z_{2}\right), D\left(z_{1}\right)\right)\right), z_{1}-z_{2}\right\rangle \\
& +\left\langle H\left(\left(A\left(z_{2}\right), B\left(z_{1}\right)\right),\left(C\left(z_{2}\right), D\left(z_{1}\right)\right)\right)\right. \\
& \left.-H\left(\left(A\left(z_{2}\right), B\left(z_{2}\right)\right),\left(C\left(z_{2}\right), D\left(z_{2}\right)\right)\right), z_{1}-z_{2}\right\rangle \\
& -\rho m\left\|z_{1}-z_{2}\right\|^{2} \\
& \geq \mu_{1}\left\|A\left(z_{1}\right)-A\left(z_{2}\right)\right\|^{2}+\gamma_{1}\left\|z_{1}-z_{2}\right\|^{2} \\
& -\mu_{2}\left\|B\left(z_{1}\right)-B\left(z_{2}\right)\right\|^{2}+\gamma_{2}\left\|z_{1}-z_{2}\right\|^{2} \\
& \text { - } \rho m\left\|z_{1}-z_{2}\right\|^{2} \\
& \geq\left[\left(\mu_{1} \alpha^{2}-\mu_{2} \beta^{2}\right)+\left(\gamma_{1}+\gamma_{2}\right)\right]\left\|z_{1}-z_{2}\right\|^{2} \\
& -\rho m\left\|z_{1}-z_{2}\right\|^{2} \\
& \geq(r-\rho m)\left\|z_{1}-z_{2}\right\|^{2} \text {, where } \\
& r=\left(\mu_{1} \alpha^{2}-\mu_{2} \beta^{2}\right)+\left(\gamma_{1}+\gamma_{2}\right),
\end{aligned}
$$

and hence

$$
\|u-v\|\left\|z_{1}-z_{2}\right\| \geq(r-\rho m)\left\|z_{1}-z_{2}\right\|^{2},
$$


that is

$$
\begin{aligned}
& \left\|R_{\rho, M}^{H((\cdot,),(\cdot, \cdot))}(u)-R_{\rho, M}^{H((\cdot,),(\cdot, \cdot))}(v)\right\| \\
& \quad \leq \frac{1}{r-\rho m}\|u-v\|, \quad \forall u, v \in X .
\end{aligned}
$$

This completes the proof.

\section{An Application of $H((\cdot, \cdot),(\cdot, \cdot))$-Mixed Cocoercive Operators for Solving Variational Inclusions}

In this section, we shall show that, under suitable assumptions, the $H((\cdot, \cdot),(\cdot, \cdot))$-mixed cocoercive operator can also play important roles for solving the generalized mixed variational inclusions in Hilbert spaces.

Let $G, S, T: X \rightarrow C B(X)$ be set-valued mappings, and let $g: X \rightarrow X, A, B, C, D: X \rightarrow X, P, F: X \times X \rightarrow X$ and $H:(X \times X) \times(X \times X) \rightarrow X$ be single-valued mappings. Suppose that $M: X \times X \rightarrow 2^{X}$ is a set-valued mapping such that for each fixed $z \in G(x), M(\cdot, z): X \rightarrow 2^{X}$ is $H((\cdot, \cdot),(\cdot, \cdot))$-mixed cocoercive operator with respect to $(A, C)$ and $(B, D)$, and $g(X) \cap \operatorname{dom}(M(\cdot, z)) \neq \emptyset$. We consider the following generalized mixed variational inclusion: for given $\omega \in X$, find $u \in X, x \in S(u), y \in T(u)$, and $z \in G(u)$ such that

$$
\omega \in F(u, z)+P(x, y)+M(g(u), z) .
$$

The problem of type (43) was studied by $\mathrm{Xu}$ and Wang [18] in the setting of Banach spaces when $M$ is $(H(\cdot, \cdot), \eta)$-monotone. Problem (43) includes many variational inequalities (inclusions) and complementarity problems as special cases as follows.

If $F=\omega=0$, then problem (43) reduces to a generalized mixed quasi-variational inclusion with $H((\cdot, \cdot),(\cdot, \cdot))$-mixed cocoercive operators in Hilbert spaces: find $u \in X, x \in S(u)$, $y \in T(u)$, and $z \in G(u)$ such that

$$
0 \in P(x, y)+M(g(u), z)
$$

If $M$ is $P-\eta$-proximal point mapping, then the problem of type (44) was studied by Kazmi and Khan [10].

If $F(u, z)=F(u), M(g(u), z)=M(u)$, and $S, T, G=0$, $P=0, g=I$, then problem (43) reduces to generalized variational inclusion problem: find $u \in X$ such that

$$
0 \in F(u)+M(u)
$$

which was studied by many authors in the setting of Hilbert spaces when $M$ is maximal monotone and $F$ is strongly monotone operator.

Lemma 14. Let $X, \omega, g, P, F, S, T, G, A, B, C, D, H$, and $M$ be the same as in problem (43). Then $(u, x, y, z)$ is a solution of problem (43) if and only if $(u, x, y, z)$ satisfies the following relation:

$$
\begin{aligned}
g(u)=R_{\rho, M}^{H((\cdot))(\cdot, \cdot))}[H((A(g(u)), B(g(u))), \\
(C(g(u)), D(g(u)))) \\
+\rho \omega-\rho P(x, y)-\rho F(u, z)],
\end{aligned}
$$

where $\rho>0$ is a constant and $R_{\rho, M}^{H((\cdot,),(\cdot,))}$ is the resolvent operator defined by (35).

Proof. Observe that, for $\rho>0$,

$$
\begin{aligned}
& \omega \in F(u, z)+P(x, y)+M(g(u), z) \\
& \Longleftrightarrow[H((A(g(u)), B(g(u))),(C(g(u)), D(g(u)))) \\
&\quad+\rho \omega-\rho P(x, y)-\rho F(u, z)] \\
& \in[H((A(g(u)), B(g(u))),(C(g(u)), D(g(u)))) \\
&\quad+\rho M(g(u), z)] \\
& \Longleftrightarrow[H((A(g(u)), B(g(u))),(C(g(u)), D(g(u)))) \\
&\quad+\rho \omega-\rho P(x, y)-\rho F(u, z)] \\
& \in(H((A, B),(C, D))+\rho M(\cdot, z)) g(u) \\
&\quad+\rho \omega-\rho P(x, y)-\rho F(u, z)] . \\
&\quad+\rho \omega-\rho P(x, y)-\rho F(u, z)] \\
& \quad[H((A(g(u)), B(g(u))),(C(g(u)), D(g(u)))) \\
& \quad
\end{aligned}
$$

Remark 15. To develop a fixed point algorithm for (43), we rewrite (46) as follows:

$$
\begin{aligned}
u=u-g(u) & \\
+R_{\rho, M(\cdot, z)}^{H((\cdot,),(\cdot,))}[H( & (A(g(u)), B(g(u))), \\
& (C(g(u)), D(g(u)))) \\
+ & \rho \omega-\rho P(x, y)-\rho F(u, z)], \\
& \rho>0 .
\end{aligned}
$$

This fixed point formulation allows us to suggest the following iterative algorithm. 
Algorithm 16.

Step 0. For given $\omega \in X$ and $\rho>0$, choose $u_{0} \in X, x_{0} \in S\left(u_{0}\right)$, $y_{0} \in T\left(u_{0}\right)$, and $z_{0} \in G\left(u_{0}\right)$. Set $n=0$.

Step 1. Let

$$
\begin{aligned}
& u_{n+1}=u_{n}-g\left(u_{n}\right) \\
&+R_{\rho, M\left(\cdot, z_{n}\right)}^{H(\cdot \cdot \cdot),(\cdot))}\left[H \left(\left(A\left(g\left(u_{n}\right)\right), B\left(g\left(u_{n}\right)\right)\right),\right.\right. \\
&\left.\left(C\left(g\left(u_{n}\right)\right), D\left(g\left(u_{n}\right)\right)\right)\right) \\
&+\left.\rho \omega-\rho P\left(x_{n}, y_{n}\right)-\rho F\left(u_{n}, z_{n}\right)\right] .
\end{aligned}
$$

Step 2. Let $u_{n+1} \in X, x_{n+1} \in S\left(u_{n+1}\right), y_{n+1} \in T\left(u_{n+1}\right)$, and $z_{n+1} \in G\left(u_{n+1}\right)$ satisfy

$$
\begin{aligned}
& x_{n} \in S\left(u_{n}\right), \\
& \left\|x_{n}-x_{n+1}\right\| \leq\left(1+\frac{1}{n+1}\right) \mathscr{D}\left(S\left(u_{n}\right), S\left(u_{n+1}\right)\right), \\
& y_{n} \in T\left(u_{n}\right), \\
& \left\|y_{n}-y_{n+1}\right\| \leq\left(1+\frac{1}{n+1}\right) \mathscr{D}\left(T\left(y_{n}\right), T\left(y_{n+1}\right)\right), \\
& z_{n} \in G\left(u_{n}\right), \\
& \left\|z_{n}-z_{n+1}\right\| \leq\left(1+\frac{1}{n+1}\right) \mathscr{D}\left(G\left(u_{n}\right), G\left(u_{n+1}\right)\right),
\end{aligned}
$$

for all $n=0,1,2, \ldots$

Step 3. If $u_{n+1}, x_{n+1}, y_{n+1}$ and $z_{n+1}$ satisfy (50) to sufficient accuracy, stop; otherwise, set $n=n+1$ and return to Step 1 .

We need the following definitions which will be used to state and prove the main result.

Definition 17. A set-valued mapping $G: X \rightarrow C B(X)$ is said to be $\mathscr{D}$-Lipschitz continuous if there exists a constant $l>0$ such that

$$
\mathscr{D}(G x, G y) \leq 1\|x-y\|, \quad \forall x, y \in X .
$$

Definition 18. Let $F: X \times X \rightarrow X$ be a single-valued mapping. Then

(i) $F$ is said to be $\delta_{1}$-Lipschitz continuous in the first argument if there exists a constant $\delta_{1}>0$ such that

$\left\|F\left(u_{1}, \cdot\right)-F\left(v_{1}, \cdot\right)\right\| \leq \delta_{1}\left\|u_{1}-v_{1}\right\|, \quad \forall u_{1}, v_{1} \in X$

(ii) $F$ is said to be $\delta_{2}$-Lipschitz continuous in the second argument if there exists a constant $\delta_{2}>0$ such that

$\left\|F\left(\cdot, u_{2}\right)-F\left(\cdot, v_{2}\right)\right\| \leq \delta_{2}\left\|u_{2}-v_{2}\right\|, \quad \forall u_{2}, v_{2} \in X$.
Definition 19. Let $P: X \times X \rightarrow X$ be a single-valued mapping. Then

(i) $P$ is said to be $\epsilon_{1}$-Lipschitz continuous in the first argument if there exists a constant $\epsilon_{1}>0$ such that

$$
\begin{array}{r}
\left\|P\left(u_{1}, v^{\prime}\right)-P\left(u_{2}, v^{\prime}\right)\right\| \leq \epsilon_{1}\left\|u_{1}-u_{2}\right\|, \\
\forall u_{1}, u_{2} \in X, v^{\prime} \in X ;
\end{array}
$$

(ii) $P$ is said to be $\epsilon_{2}$-Lipschitz continuous in the second argument if there exists a constant $\epsilon_{2}>0$ such that

$$
\begin{array}{r}
\left\|P\left(u^{\prime}, v_{1}\right)-P\left(u^{\prime}, v_{2}\right)\right\| \leq \epsilon_{2}\left\|v_{1}-v_{2}\right\|, \\
\forall v_{1}, v_{2} \in X, u^{\prime} \in X .
\end{array}
$$

Theorem 20. Let $S, T, G: X \rightarrow C B(X)$ be set-valued mappings, let $g, A, B, C, D: X \rightarrow X, P, F: X \times X \rightarrow X$ and $H:(X \times X) \times(X \times X) \rightarrow X$ be single-valued mappings, and let $M: X \times X \rightarrow 2^{X}$ be a set-valued mapping such that for each $z \in G(x), M(\cdot, z): X \rightarrow 2^{X}$ is $H((\cdot, \cdot),(\cdot, \cdot))$ mixed cocoercive operator with respect to $(A, C)$ and $(B, D)$, and $g(X) \cap \operatorname{dom}(\mathrm{M}(\cdot, \mathrm{z})) \neq \emptyset$. Assume that

(i) S, T, and $G$ are D-Lipschitz continuous with constants $l_{1}, l_{2}$ and $l_{3}$, respectively;

(ii) $A$ is $\alpha$-expansive, and $B$ is $\beta$-Lipschitz continuous;

(iii) $H((A, B),(C, D))$ is $\tau$-mixed Lipschitz continuous with constant $\tau>0$;

(iv) $g$ is $\zeta$-Lipschitz continuous and $\xi$-strongly monotone;

(v) $P$ is $\epsilon_{1}$-Lipschitz continuous in the first argument and $\epsilon_{2}$-Lipschitz continuous in the second argument;

(vi) $F$ is $\delta_{1}$-Lipschitz continuous in the first argument and $\delta_{2}$-Lipschitz continuous in the second argument;

(vii) $H((A, B),(C, D))$ is v-mixed strongly monotone with respect to $P, S$, and $T$ with constant $v>0$;

(viii) Suppose that there exist constants $\kappa>0, \rho>0$ such that, for each $u \in X$ and $(z, \bar{z}) \in X \times X$,

$$
\begin{gathered}
\left\|R_{\rho, M(\cdot, z)}^{H((\cdot),(\cdot,))}(u)-R_{\rho, M(\cdot, \cdot \bar{z})}^{H((\cdot))(\cdot,))}(u)\right\|=\kappa\|z-\bar{z}\| ; \\
0<\sqrt{1-2 \xi+\zeta^{2}}+\kappa l_{3} \\
+\left(\left\{\sqrt{\tau^{2} \zeta^{2}-2 \rho \xi v+\rho^{2}\left(\epsilon_{1} l_{1}+\epsilon_{2} l_{2}\right)^{2}}\right.\right. \\
\left.\left.\quad+\rho\left(\delta_{1}+\delta_{2} l_{3}\right)\right\}\right)(r-\rho m)^{-1}<1,
\end{gathered}
$$

where $r=\left(\mu_{1} \alpha^{2}-\mu_{2} \beta^{2}\right)+\left(\gamma_{1}+\gamma_{2}\right)>\rho m$ and $\mu_{1}>$ $\mu_{2}, \alpha>\beta, \gamma_{1}, \gamma_{2}>0$.

Then generalized mixed variational inclusion problem (43) has a solution $(u, x, y, z)$, where $u \in X, x \in S(u), y \in T(u)$, and $z \in G(u)$, and the iterative sequences $\left\{u_{n}\right\},\left\{x_{n}\right\},\left\{y_{n}\right\},\left\{z_{n}\right\}$, generated by Algorithm 16 converges strongly to $u, x, y$, and $z$, respectively. 
Proof. Since $S, T$, and $G$ are $\mathscr{D}$-Lipschitz continuous with constants $l_{1}, l_{2}$, and $l_{3}$, respectively, it follows from Algorithm 16 that

$$
\begin{aligned}
\left\|x_{n+1}-x_{n}\right\| & \leq\left(1+\frac{1}{n+1}\right) \mathscr{D}\left(S\left(u_{n+1}\right), S\left(u_{n}\right)\right) \\
& \leq\left(1+\frac{1}{n+1}\right) \mathfrak{1}_{1}\left\|u_{n+1}-u_{n}\right\|, \\
\left\|y_{n+1}-y_{n}\right\| & \leq\left(1+\frac{1}{n+1}\right) \mathscr{D}\left(T\left(u_{n+1}\right), T\left(u_{n}\right)\right) \\
& \leq\left(1+\frac{1}{n+1}\right) \mathfrak{l}_{2}\left\|u_{n+1}-u_{n}\right\|, \\
\left\|z_{n+1}-z_{n}\right\| & \leq\left(1+\frac{1}{n+1}\right) \mathscr{D}\left(G\left(u_{n+1}\right), G\left(u_{n}\right)\right) \\
& \leq\left(1+\frac{1}{n+1}\right) \mathfrak{x}_{3}\left\|u_{n+1}-u_{n}\right\|,
\end{aligned}
$$

for $n=0,1,2, \ldots$.

Now, we estimate $\left\|u_{n}-u_{n-1}-\left(g\left(u_{n}\right)-g\left(u_{n-1}\right)\right)\right\|$, by Lipschitz continuity and strong monotonicity of $g$, we have

$$
\begin{aligned}
\| u_{n} & -u_{n-1}-\left(g\left(u_{n}\right)-g\left(u_{n-1}\right)\right) \|^{2} \\
= & \left\|u_{n}-u_{n-1}\right\|^{2}-2\left\langle g\left(u_{n}\right)-g\left(u_{n-1}\right), u_{n}-u_{n-1}\right\rangle \\
& +\left\|g\left(u_{n}\right)-g\left(u_{n-1}\right)\right\|^{2} \\
\leq & \left\|u_{n}-u_{n-1}\right\|^{2}-2 \xi\left\|u_{n}-u_{n-1}\right\|^{2}+\zeta^{2}\left\|u_{n}-u_{n-1}\right\|^{2} \\
= & \left(1-2 \xi+\zeta^{2}\right)\left\|u_{n}-u_{n-1}\right\|^{2} .
\end{aligned}
$$

Now, we estimate $\left\|u_{n+1}-u_{n}\right\|$ by using Algorithm 16 and the Lipschitz continuity of $R_{\rho, M\left(\cdot, \cdot z_{n}\right)}^{H((\cdot, \cdot))}$ :

$$
\begin{aligned}
& \left\|u_{n+1}-u_{n}\right\| \\
& =\| u_{n}-g\left(u_{n}\right) \\
& +R_{\rho, M\left(\cdot, z_{n}\right)}^{H((\cdot), \cdot(\cdot))}\left[H \left(\left(A\left(g\left(u_{n}\right)\right), B\left(g\left(u_{n}\right)\right)\right),\right.\right. \\
& \left.\quad\left(C\left(g\left(u_{n}\right)\right), D\left(g\left(u_{n}\right)\right)\right)\right) \\
& \left.+\rho \omega-\rho P\left(x_{n}, y_{n}\right)-\rho F\left(u_{n}, z_{n}\right)\right] \\
& -\left[u_{n-1}-g\left(u_{n-1}\right)\right. \\
& +R_{\rho, M\left(\cdot, \cdot z_{n-1}\right)}^{H((\cdot \cdot),(\cdot))}\left[H \left(\left(A\left(g\left(u_{n-1}\right)\right), B\left(g\left(u_{n-1}\right)\right)\right),\right.\right. \\
& \left.\quad\left(C\left(g\left(u_{n-1}\right)\right), D\left(g\left(u_{n-1}\right)\right)\right)\right) \\
& +\rho \omega-\rho P\left(x_{n-1}, y_{n-1}\right) \\
& \left.\left.-\rho F\left(u_{n-1}, z_{n-1}\right)\right]\right] \|
\end{aligned}
$$

$$
\begin{aligned}
& \leq\left\|u_{n}-u_{n-1}-\left(g\left(u_{n}\right)-g\left(u_{n-1}\right)\right)\right\| \\
& +\| R_{\rho, M\left(\cdot, z_{n}\right)}^{H(\cdot \cdot),(\cdot))}\left[H \left(\left(A\left(g\left(u_{n}\right)\right), B\left(g\left(u_{n}\right)\right)\right),\right.\right. \\
& \left.\left(C\left(g\left(u_{n}\right)\right), D\left(g\left(u_{n}\right)\right)\right)\right) \\
& \left.+\rho \omega-\rho P\left(x_{n}, y_{n}\right)-\rho F\left(u_{n}, z_{n}\right)\right] \\
& -\left[R _ { \rho , M ( \cdot , \cdot z _ { n - 1 } ) } ^ { H ( \cdot , \cdot ( \cdot ) ) } \left[H \left(\left(A\left(g\left(u_{n-1}\right)\right), B\left(g\left(u_{n-1}\right)\right)\right)\right.\right.\right. \text {, } \\
& \left.\left(C\left(g\left(u_{n-1}\right)\right), D\left(g\left(u_{n-1}\right)\right)\right)\right) \\
& +\rho \omega-\rho P\left(x_{n-1}, y_{n-1}\right) \\
& \left.\left.-\rho F\left(u_{n-1}, z_{n-1}\right)\right]\right] \| \\
& \leq\left\|u_{n}-u_{n-1}-\left(g\left(u_{n}\right)-g\left(u_{n-1}\right)\right)\right\| \\
& +\| R_{\rho, M\left(\cdot, z_{n}\right)}^{H(\cdot \cdot \cdot)(\cdot, \cdot))}\left[H \left(\left(A\left(g\left(u_{n}\right)\right), B\left(g\left(u_{n}\right)\right)\right),\right.\right. \\
& \left.\left(C\left(g\left(u_{n}\right)\right), D\left(g\left(u_{n}\right)\right)\right)\right) \\
& \left.+\rho \omega-\rho P\left(x_{n}, y_{n}\right)-\rho F\left(u_{n}, z_{n}\right)\right] \\
& -R_{\rho, M\left(\cdot, z_{n}\right)}^{H((\cdot,))(\cdot, \cdot)}\left[H \left(\left(A\left(g\left(u_{n-1}\right)\right), B\left(g\left(u_{n-1}\right)\right)\right)\right.\right. \text {, } \\
& \left.\left(C\left(g\left(u_{n-1}\right)\right), D\left(g\left(u_{n-1}\right)\right)\right)\right) \\
& +\rho \omega-\rho P\left(x_{n-1}, y_{n-1}\right) \\
& \left.-\rho F\left(u_{n-1}, z_{n-1}\right)\right] \| \\
& +\| R_{\rho, M\left(\cdot, z_{n}\right)}^{H((\cdot,))(\cdot))}\left[H \left(\left(A\left(g\left(u_{n-1}\right)\right), B\left(g\left(u_{n-1}\right)\right)\right),\right.\right. \\
& \left.\left(C\left(g\left(u_{n-1}\right)\right), D\left(g\left(u_{n-1}\right)\right)\right)\right) \\
& +\rho \omega-\rho P\left(x_{n-1}, y_{n-1}\right) \\
& \left.-\rho F\left(u_{n-1}, z_{n-1}\right)\right] \\
& -R_{\rho, M\left(\cdot, z_{n-1}\right)}^{H((\cdot, \cdot)(\cdot,))}\left[H \left(\left(A\left(g\left(u_{n-1}\right)\right), B\left(g\left(u_{n-1}\right)\right)\right)\right.\right. \text {, } \\
& \left.\left(C\left(g\left(u_{n-1}\right)\right), D\left(g\left(u_{n-1}\right)\right)\right)\right) \\
& +\rho \omega-\rho P\left(x_{n-1}, y_{n-1}\right) \\
& \left.-\rho F\left(u_{n-1}, z_{n-1}\right)\right] \| \\
& \leq\left\|u_{n}-u_{n-1}-\left(g\left(u_{n}\right)-g\left(u_{n-1}\right)\right)\right\|+\frac{1}{r-\rho m} \\
& \times\left[\| H\left(\left(A\left(g\left(u_{n}\right)\right), B\left(g\left(u_{n}\right)\right)\right),\right.\right. \\
& \left.\left(C\left(g\left(u_{n}\right)\right), D\left(g\left(u_{n}\right)\right)\right)\right) \\
& -H\left(\left(A\left(g\left(u_{n-1}\right)\right), B\left(g\left(u_{n-1}\right)\right)\right)\right. \text {, } \\
& \left.\left(C\left(g\left(u_{n-1}\right)\right), D\left(g\left(u_{n-1}\right)\right)\right)\right)
\end{aligned}
$$




$$
\begin{aligned}
& -\rho\left(P\left(x_{n}, y_{n}\right)-P\left(x_{n-1}, y_{n-1}\right)\right) \| \\
& \left.+\rho\left\|F\left(u_{n}, z_{n}\right)-F\left(u_{n-1}, z_{n-1}\right)\right\|\right]+\kappa\left\|z_{n}-z_{n-1}\right\| .
\end{aligned}
$$

Now, by Algorithm 16 and conditions (i), (vi), and (vii), we get

$$
\begin{aligned}
&\left\|P\left(x_{n}, y_{n}\right)-P\left(x_{n-1}, y_{n-1}\right)\right\| \\
& \leq\left\|P\left(x_{n}, y_{n}\right)-P\left(x_{n-1}, y_{n}\right)\right\| \\
&+\left\|P\left(x_{n-1}, y_{n}\right)-P\left(x_{n-1}, y_{n-1}\right)\right\| \\
& \leq \epsilon_{1}\left\|x_{n}-x_{n-1}\right\|+\epsilon_{2}\left\|y_{n}-y_{n-1}\right\| \\
& \leq \epsilon_{1}\left(1+\frac{1}{n}\right) \mathscr{D}\left(S\left(u_{n}\right), S\left(u_{n-1}\right)\right) \\
&+\epsilon_{2}\left(1+\frac{1}{n}\right) \mathscr{D}\left(T\left(u_{n}\right), T\left(u_{n-1}\right)\right) \\
& \leq \epsilon_{1}\left(1+\frac{1}{n}\right) l_{1}\left\|u_{n}-u_{n-1}\right\| \\
&+\epsilon_{2}\left(1+\frac{1}{n}\right) l_{2}\left\|u_{n}-u_{n-1}\right\| \\
& \leq\left(\epsilon_{1} l_{1}+\epsilon_{2} l_{2}\right)\left(1+\frac{1}{n}\right)\left\|u_{n}-u_{n-1}\right\| \\
& \leq F(\left(u_{n}, z_{n}\right)-F\left(u_{n-1}, z_{n-1}\right) \| \\
& \leq\left\|F\left(u_{n}, z_{n}\right)-F\left(u_{n-1}, z_{n}\right)\right\| \\
&+\left\|F\left(u_{n-1}, z_{n}\right)-F\left(u_{n-1}, z_{n-1}\right)\right\| \\
& \leq \delta_{1}\left\|u_{n}-u_{n-1}\right\|+\delta_{2}\left\|z_{n}-z_{n-1}\right\| \\
&+u_{n}-u_{n-1} \| \\
&\left.+1+\frac{1}{n}\right) \mathscr{D}\left(G\left(u_{n}\right), G\left(u_{n-1}\right)\right) \\
& \\
&
\end{aligned}
$$

Since $H((A, B),(C, D))$ is $\tau$-mixed Lipschitz continuous and $v$-mixed strongly monotone with respect to $P, S$, and $T$ and $g$ is $\xi$-Lipschitz continuous and from (61), we have

$$
\begin{gathered}
\| H\left(\left(A\left(g\left(u_{n}\right)\right), B\left(g\left(u_{n}\right)\right)\right),\left(C\left(g\left(u_{n}\right)\right), D\left(g\left(u_{n}\right)\right)\right)\right) \\
-H\left(\left(A\left(g\left(u_{n-1}\right)\right), B\left(g\left(u_{n-1}\right)\right)\right),\right. \\
\left.\quad\left(C\left(g\left(u_{n-1}\right)\right), D\left(g\left(u_{n-1}\right)\right)\right)\right) \\
-\rho\left(P\left(x_{n}, y_{n}\right)-P\left(x_{n-1}, y_{n-1}\right)\right) \|^{2} \\
\leq \| H\left(\left(A\left(g\left(u_{n}\right)\right), B\left(g\left(u_{n}\right)\right)\right),\left(C\left(g\left(u_{n}\right)\right), D\left(g\left(u_{n}\right)\right)\right)\right) \\
-H\left(\left(A\left(g\left(u_{n-1}\right)\right), B\left(g\left(u_{n-1}\right)\right)\right),\right. \\
\left.\left(C\left(g\left(u_{n-1}\right)\right), D\left(g\left(u_{n-1}\right)\right)\right)\right) \|^{2}
\end{gathered}
$$$$
-2 \rho\left\langleH \left(\left(A\left(g\left(u_{n}\right)\right), B\left(g\left(u_{n}\right)\right)\right),\right.\right.
$$$$
\left.\left(C\left(g\left(u_{n}\right)\right), D\left(g\left(u_{n}\right)\right)\right)\right)
$$$$
\text { - H ((A } \left.\left(g\left(u_{n-1}\right)\right), B\left(g\left(u_{n-1}\right)\right)\right) \text {, }
$$$$
\left.\left(C\left(g\left(u_{n-1}\right)\right), D\left(g\left(u_{n-1}\right)\right)\right)\right) \text {, }
$$$$
\left.-\rho\left(P\left(x_{n}, y_{n}\right)-P\left(x_{n-1}, y_{n-1}\right)\right)\right\rangle
$$$$
+\rho^{2}\left\|P\left(x_{n}, y_{n}\right)-P\left(x_{n-1}, y_{n-1}\right)\right\|^{2}
$$$$
\leq \tau^{2}\left\|g\left(u_{n}\right)-g\left(u_{n-1}\right)\right\|^{2}-2 \rho \xi v\left\|u_{n}-u_{n-1}\right\|^{2}
$$$$
+\rho^{2}\left(\epsilon_{1} l_{1}+\epsilon_{2} l_{2}\right)^{2}\left(1+\frac{1}{n}\right)^{2}\left\|u_{n}-u_{n-1}\right\|^{2}
$$$$
\leq\left(\tau^{2} \zeta^{2}-2 \rho \xi v+\rho^{2}\left(\epsilon_{1} l_{1}+\epsilon_{2} l_{2}\right)^{2}\left(1+\frac{1}{n}\right)^{2}\right)
$$$$
\times\left\|u_{n}-u_{n-1}\right\|^{2} .
$$

Using (59)-(63), we have

$$
\begin{aligned}
&\left\|u_{n+1}-u_{n}\right\| \sqrt{1-2 \xi+\zeta^{2}}\left\|u_{n}-u_{n-1}\right\|+\frac{1}{r-\rho m} \\
& \leq {\left[\sqrt{\tau^{2} \zeta^{2}-2 \rho \xi v+\rho^{2}\left(\epsilon_{1} l_{1}+\epsilon_{2} l_{2}\right)^{2}\left(1+\frac{1}{n}\right)^{2}}\right.} \\
& \times\left\|u_{n}-u_{n-1}\right\| \\
&\left.+\rho\left(\delta_{1}+\left(1+\frac{1}{n}\right) \delta_{2} l_{3}\right)\left\|u_{n}-u_{n-1}\right\|\right] \\
&+ \kappa\left(1+\frac{1}{n}\right) l_{3}\left\|u_{n}-u_{n-1}\right\| \\
&=\left.\sqrt{1-2 \xi+\zeta^{2}}+\kappa\left(1+\frac{1}{n}\right) l_{3}\right]\left\|u_{n}-u_{n-1}\right\| \\
&+\left(\left\{\sqrt{\tau^{2} \zeta^{2}-2 \rho \xi v+\rho^{2}\left(\epsilon_{1} l_{1}+\epsilon_{2} l_{2}\right)^{2}\left(1+\frac{1}{n}\right)^{2}}\right.\right. \\
&\left.\left.+\rho\left(\delta_{1}+\left(1+\frac{1}{n}\right) \delta_{2} l_{3}\right)\right\}\right)(r-\rho m)^{-1}
\end{aligned}
$$$$
\times\left\|u_{n}-u_{n-1}\right\|
$$$$
=\left[\sqrt{1-2 \xi+\zeta^{2}}+\kappa\left(1+\frac{1}{n}\right) l_{3}\right.
$$$$
+\left(\left\{\sqrt{\tau^{2} \zeta^{2}-2 \rho \xi v+\rho^{2}\left(\epsilon_{1} l_{1}+\epsilon_{2} l_{2}\right)^{2}\left(1+\frac{1}{n}\right)^{2}}\right.\right.
$$$$
\left.\left.\left.+\rho\left(\delta_{1}+\left(1+\frac{1}{n}\right) \delta_{2} l_{3}\right)\right\}\right)(r-\rho m)^{-1}\right]
$$

$\times\left\|u_{n}-u_{n-1}\right\|$, 
which implies

$$
\left\|u_{n+1}-u_{n}\right\| \leq \Lambda_{n}\left\|u_{n}-u_{n-1}\right\|,
$$

where

$$
\begin{aligned}
\Lambda_{n}= & \sqrt{1-2 \xi+\zeta^{2}}+\kappa\left(1+\frac{1}{n}\right) l_{3} \\
+( & \left\{\sqrt{\tau^{2} \zeta^{2}-2 \rho \xi v+\rho^{2}\left(\epsilon_{1} l_{1}+\epsilon_{2} l_{2}\right)^{2}\left(1+\frac{1}{n}\right)^{2}}\right. \\
& \left.\left.+\rho\left(\delta_{1}+\left(1+\frac{1}{n}\right) \delta_{2} l_{3}\right)\right\}\right)(r-\rho m)^{-1}
\end{aligned}
$$

Let

$$
\begin{aligned}
\Lambda= & \sqrt{1-2 \xi+\zeta^{2}}+\kappa l_{3} \\
& +\frac{\left\{\sqrt{\tau^{2} \zeta^{2}-2 \rho \xi v+\rho^{2}\left(\epsilon_{1} l_{1}+\epsilon_{2} l_{2}\right)^{2}}+\rho\left(\delta_{1}+\delta_{2} l_{3}\right)\right\}}{r-\rho m} .
\end{aligned}
$$

Since $n \rightarrow \infty$, so $\Lambda_{n} \rightarrow \Lambda$. By assumption (ix), it is easy to see that $\Lambda<1$. Therefore, (67) implies that $\left\{u_{n}\right\}$ is a Cauchy sequence in $X$. Since $X$ is a Hilbert space, there exists $u \in X$ such that $u_{n} \rightarrow u$ as $n \rightarrow \infty$. From (58), we know that $\left\{x_{n}\right\},\left\{y_{n}\right\}$, and $\left\{z_{n}\right\}$ are also Cauchy sequences in $X$; thus, there exists $x, y, z \in X$ such that $x_{n} \rightarrow x, y_{n} \rightarrow y$, and $z_{n} \rightarrow z$ as $n \rightarrow \infty$, respectively. By the continuity of $g, R_{\rho, M(\cdot, z)}^{H(\cdot \cdot),(\cdot \cdot))}, A, B, C, D, S, T$, and $G$ and Algorithm 16, we have

$$
\begin{aligned}
& g(u)= R_{\rho, M(\cdot, z)}^{H((\cdot,),(\cdot,))} \\
& \times[H((A(g(u)), B(g(u))),(C(g(u)), D(g(u)))) \\
&\quad-\rho \omega-\rho P(x, y)-\rho F(u, z)] .
\end{aligned}
$$
have

Now, we prove that $x \in S(u)$. In fact, since $x_{n} \in S\left(u_{n}\right)$, we

$$
\begin{aligned}
d(x, S(u)) & \leq\left\|x-x_{n}\right\|+d\left(x_{n}, S(u)\right) \\
& \leq\left\|x-x_{n}\right\|+\mathscr{D}\left(S\left(u_{n}\right), S(u)\right) \\
& \leq\left\|x-x_{n}\right\|+\rho\left\|x_{n}-x\right\| \longrightarrow 0 \text { as } n \longrightarrow \infty,
\end{aligned}
$$

which implies that $d(x, S(u))=0$. Since $S(u) \in C B(X)$, it follows that $x \in S(u)$. Similarly, it is easy to see that $y \in$ $T(u), z \in G(u)$. By Lemma 14, $(u, x, y, z)$ is the solution of problem (43).
Example 21. Let $X=\mathbb{R}^{2}$ with usual inner product.

Let $S, T, G: \mathbb{R}^{2} \rightarrow 2^{\mathbb{R}^{2}}$ be defined by

$$
S(x)=T(x)=G(x)
$$

$$
=\left\{\left(\begin{array}{l}
\frac{1}{n} x_{1} \\
\frac{1}{n} x_{2}
\end{array}\right), \forall n \in N, x=\left(x_{1}, x_{2}\right) \in \mathbb{R}^{2}\right\} .
$$

Then, it is easy to check that

(i) $S, T$, and $G$ are (1/10) D-Lipschitz continuous for $n=$ 10.

Let $A, B, C, D: \mathbb{R}^{2} \rightarrow \mathbb{R}^{2}$ be defined by

$$
\begin{array}{r}
A x=\left(\begin{array}{c}
\frac{1}{10} x_{1} \\
\frac{1}{10} x_{2}
\end{array}\right), \quad B y=\left(\begin{array}{c}
-\frac{1}{5} y_{1} \\
-\frac{1}{5} y_{2}
\end{array}\right), \\
C x=\left(\begin{array}{l}
2 x_{1} \\
2 x_{2}
\end{array}\right), \quad D y=\left(\begin{array}{l}
y_{1} \\
y_{2}
\end{array}\right), \\
\forall x=\left(x_{1}, x_{2}\right), \quad y=\left(y_{1}, y_{2}\right) \in \mathbb{R}^{2} .
\end{array}
$$

Suppose that $H:\left(\mathbb{R}^{2} \times \mathbb{R}^{2}\right) \times\left(\mathbb{R}^{2} \times \mathbb{R}^{2}\right) \rightarrow \mathbb{R}^{2}$ is defined by

$H((A x, B y),(C x, D y))=A x+B y+C x+D y, \quad \forall x \in \mathbb{R}^{2}$.

Then it is easy to cheek that

(ii) $H((\cdot, \cdot),(\cdot, \cdot))$ is $(10,2)$-mixed cocoercive with respect to $(A, C)$ and $(5,1)$-relaxed mixed cocoercive with respect to $(B, D), A$ is $(1 / n)$-expansive for $n=10,11$, and $B$ is $(1 / n)$-Lipschitz continuous for $n=4,5$;

(iii) $H((A, B),(C, D))$ is $(29 / n)$-mixed Lipschitz continuous with respect to $A, B, C$, and $D$ for $n=9,10$.

Let $g: \mathbb{R}^{2} \rightarrow \mathbb{R}^{2}$ be defined by

$$
g(x)=\left(\begin{array}{c}
\frac{9}{10} x_{1} \\
\frac{9}{10} x_{2}
\end{array}\right), \quad \forall x \in \mathbb{R}^{2} .
$$

Then it is easy to check that

(iv) $g$ is $(9 / n)$-strongly monotone for $n=10,11$ and $(9 / n)$ Lipschitz continuous for $n=9,10$.

Let $P, F: \mathbb{R}^{2} \times \mathbb{R}^{2} \rightarrow \mathbb{R}^{2}$ be defined by

$$
P(x, y)=\frac{x}{4}+\frac{y}{5}, \quad F(x, y)=\frac{x}{5}+\frac{y}{4}, \quad \forall x, y \in \mathbb{R}^{2} .
$$


Then it is easy to check that

(v) $P$ is $(1 / n)$-Lipschitz continuous in the first argument for $n=3,4$ and $(1 / n)$-Lipschitz continuous in the second argument for $n=4,5$;

(vi) $F$ is $(1 / n)$-Lipschitz continuous in the first argument for $n=4,5$ and $(1 / n)$-Lipschitz continuous in the second argument for $n=3,4$;

(vii) $H((A, B),(C, D))$ is $(135 / n)$-mixed strongly monotone with respect to $P, S$, and $T$ for $n=999,1000$.

In addition, Let $M: \mathbb{R}^{2} \times \mathbb{R}^{2} \rightarrow 2^{\mathbb{R}^{2}}$ be defined by

$$
M(x, z)=-2 x+z, \quad M(x, \bar{z})=-\frac{9}{10} x+\bar{z},
$$

where $z=\left(-(9 / 10) x_{1},-(9 / 10) x_{2}\right), \bar{z}=\left(-2 x_{1},-2 x_{2}\right)$ $\forall x, z, \bar{z} \in \mathbb{R}^{2}$.

Then it is easy to check that

(viii)

$$
\begin{array}{r}
\left\|R_{\rho, M(\cdot, z)}^{H((\cdot),(\cdot))}(u)-R_{\rho, M(\cdot, \bar{z})}^{H((\cdot \cdot),(\cdot))}(u)\right\| \leq \frac{9}{n}\|z-\bar{z}\|, \\
\text { for } n=28,29 .
\end{array}
$$

Therefore, for the constants

$$
\begin{gathered}
l_{1}=l_{2}=l_{3}=\frac{1}{10}, \\
\mu_{1}=10, \quad \gamma_{1}=2, \quad \mu_{2}=5, \\
\gamma_{2}=1, \quad \epsilon_{1}=\frac{1}{4}, \quad \epsilon_{2}=\frac{1}{5}, \\
\delta_{1}=\frac{1}{5}, \quad \delta_{2}=\frac{1}{4}, \quad \alpha=\frac{1}{10}, \\
\beta=\frac{1}{5}, \quad \zeta=\frac{9}{10}, \quad \xi=\frac{9}{10}, \\
m=2.9, \quad \kappa=\frac{9}{29}, \quad \rho=0.01, \quad r=2.9,
\end{gathered}
$$

obtained in (i) to (viii), all the conditions of Theorem 20 are satisfied for the generalized mixed variational inclusion problem (43) for $\rho=0.01$.

Remark 22. If the set-valued mapping $M$ is $\eta$-monotone, $\eta(x, y)$ is Lipschitz continuous, then $H((\cdot, \cdot),(\cdot, \cdot))$ becomes a new $(H((\cdot, \cdot),(\cdot, \cdot)), \eta)$ monotone operator; see, for example, [18]. We leave the proofs to readers who are interested in this area.

\section{Acknowledgment}

The authors are extremely grateful to the referee for the useful suggestions that improved the content of the paper.

\section{References}

[1] D. Aussel and N. Hadjisavvas, "On quasimonotone variational inequalities," Journal of Optimization Theory and Applications, vol. 121, no. 2, pp. 445-450, 2004.

[2] N. Hadjisavvas, "Continuity and maximality properties of pseudomonotone operators," Journal of Convex Analysis, vol. 10, no. 2, pp. 465-475, 2003.

[3] N. Hadjisavvas and S. Schaible, "On a generalization of paramonotone maps and its application to solving the Stampacchia variational inequality," Optimization, vol. 55, no. 5-6, pp. 593604, 2006.

[4] N. Hadjisavvas, S. Schaible, and N.-C. Wong, "Pseudomonotone operators: a survey of the theory and its applications," Journal of Optimization Theory and Applications, vol. 152, no. 1, pp. 1-20, 2012.

[5] P. Tseng, "Further applications of a splitting algorithm to decomposition in variational inequalities and convex programming," Mathematical Programming, vol. 48, no. 2, pp. 249-263, 1990.

[6] T. L. Magnanti and G. Perakis, "Convergence condition for variational inequality algorithms," Working Paper OR-282-93, Massachusetts Institute of Technology, 1993.

[7] D. L. Zhu and P. Marcotte, "Co-coercivity and its role in the convergence of iterative schemes for solving variational inequalities," SIAM Journal on Optimization, vol. 6, no. 3, pp. 714-726, 1996.

[8] Y.-P. Fang and N.-J. Huang, " $H$-monotone operator and resolvent operator technique for variational inclusions," Applied Mathematics and Computation, vol. 145, no. 2-3, pp. 795-803, 2003.

[9] Y.-P. Fang and N.-J. Huang, " $H$-accretive operators and resolvent operator technique for solving variational inclusions in Banach spaces," Applied Mathematics Letters, vol. 17, no. 6, pp. 647-653, 2004.

[10] K. R. Kazmi and F. A. Khan, "Iterative approximation of a solution of multi-valued variational-like inclusion in Banach spaces: a $P-\eta$-proximal-point mapping approach," Journal of Mathematical Analysis and Applications, vol. 325, no. 1, pp. 665674, 2007.

[11] K. R. Kazmi and F. A. Khan, "Sensitivity analysis for parametric generalized implicit quasi-variational-like inclusions involving $P$ - $\eta$-accretive mappings," Journal of Mathematical Analysis and Applications, vol. 337, no. 2, pp. 1198-1210, 2008.

[12] Y.-P. Fang, N.-J. Huang, and H. B. Thompson, "A new system of variational inclusions with $(H, \eta)$-monotone operators in Hilbert spaces," Computers \& Mathematics with Applications, vol. 49, no. 2-3, pp. 365-374, 2005.

[13] H.-Y. Lan, Y. J. Cho, and R. U. Verma, "Nonlinear relaxed cocoercive variational inclusions involving $(A, \eta)$-accretive mappings in Banach spaces," Computers \& Mathematics with Applications, vol. 51, no. 9-10, pp. 1529-1538, 2006.

[14] Y.-Z. Zou and N.-J. Huang, " $H(\cdot, \cdot)$-accretive operator with an application for solving variational inclusions in Banach spaces," Applied Mathematics and Computation, vol. 204, no. 2, pp. 809816, 2008.

[15] K. R. Kazmi, N. Ahmad, and M. Shahzad, "Convergence and stability of an iterative algorithm for a system of generalized implicit variational-like inclusions in Banach spaces," Applied Mathematics and Computation, vol. 218, no. 18, pp. 9208-9219, 2012. 
[16] K. R. Kazmi, M. I. Bhat, and N. Ahmad, "An iterative algorithm based on $M$-proximal mappings for a system of generalized implicit variational inclusions in Banach spaces," Journal of Computational and Applied Mathematics, vol. 233, no. 2, pp. 361-371, 2009.

[17] K. R. Kazmi, F. A. Khan, and M. Shahzad, "A system of generalized variational inclusions involving generalized $H(\cdot, \cdot)$ accretive mapping in real q-uniformly smooth Banach spaces," Applied Mathematics and Computation, vol. 217, no. 23, pp. 9679-9688, 2011.

[18] Z. Xu and Z. Wang, "A generalized mixed variational inclusion involving $(H(\cdot, \cdot), \eta)$-monotone operators in Banach spaces," Journal of Mathematics Research, vol. 2, no. 3, pp. 47-56, 2010.

[19] R. Ahmad, M. Dilshad, M.-M. Wong, and J.-C. Yao, “ $H(\cdot, \cdot)$ cocoercive operator and an application for solving generalized variational inclusions," Abstract and Applied Analysis, vol. 2011, Article ID 261534, 12 pages, 2011.

[20] S. Husain, S. Gupta, and V. N. Mishra, "Graph convergence for the $H(\cdot, \cdot)$-mixed mapping with an application for solving the system of generalized variational inclusions," Fixed Point Theory and Applications, vol. 2013, article 304, 2013.

[21] S. Husain, S. Gupta, and V. N. Mishra, "Generalized $H(\cdot, \cdot, \cdot)-\eta$ cocoercive operators and generalized set-valued VariationalLike Inclusions," Journal of Mathematics, vol. 2013, Article ID 738491, 10 pages, 2013.

[22] S. Husain and S. Gupta, "Algorithm for solving a new system of generalized variational inclusions in Hilbert spaces," Journal of Calculus of Variations, vol. 2013, Article ID 461371, 8 pages, 2013. 


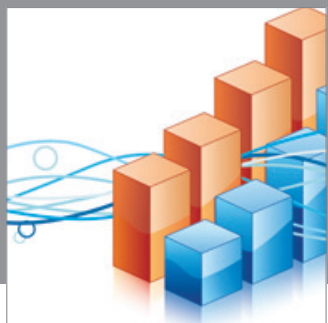

Advances in

Operations Research

mansans

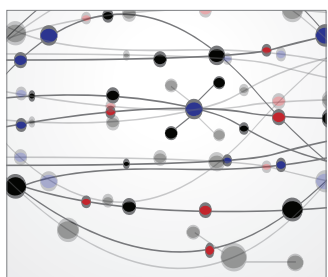

The Scientific World Journal
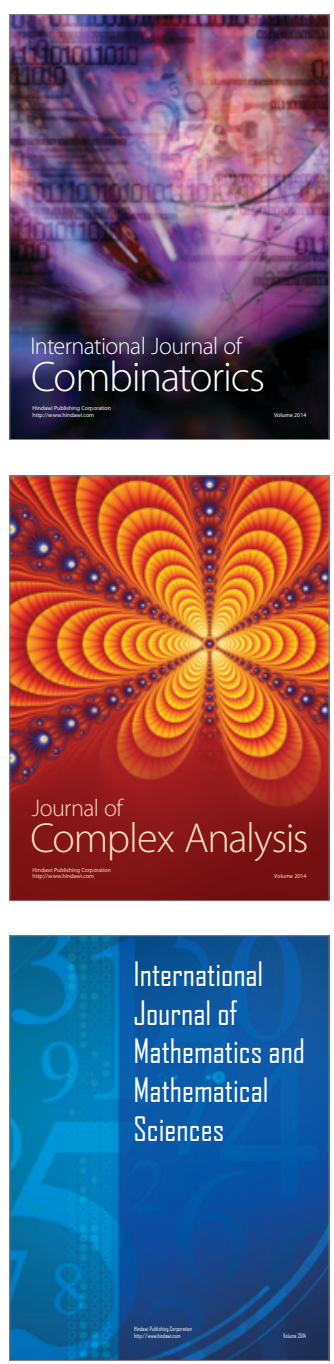
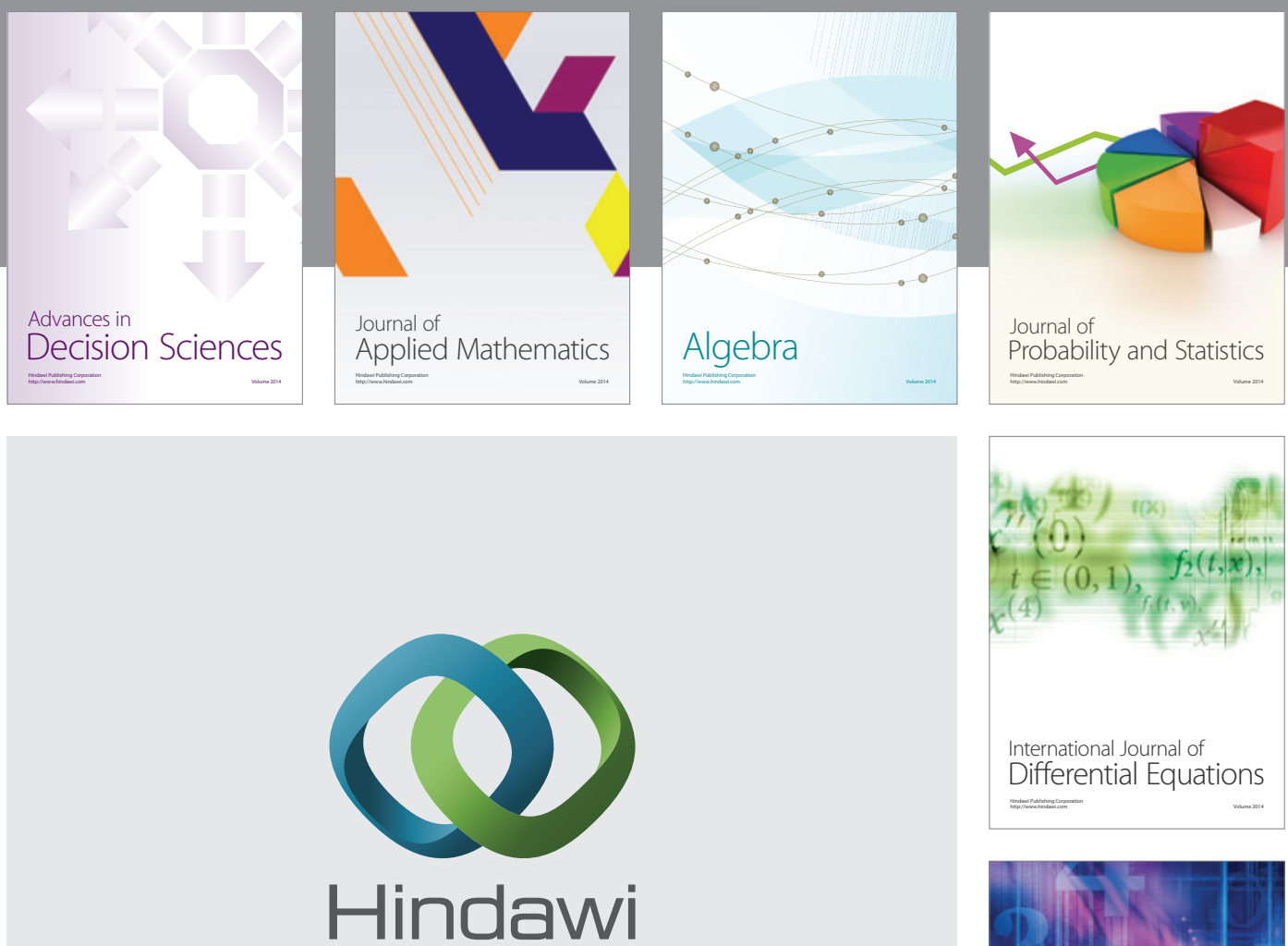

Submit your manuscripts at http://www.hindawi.com
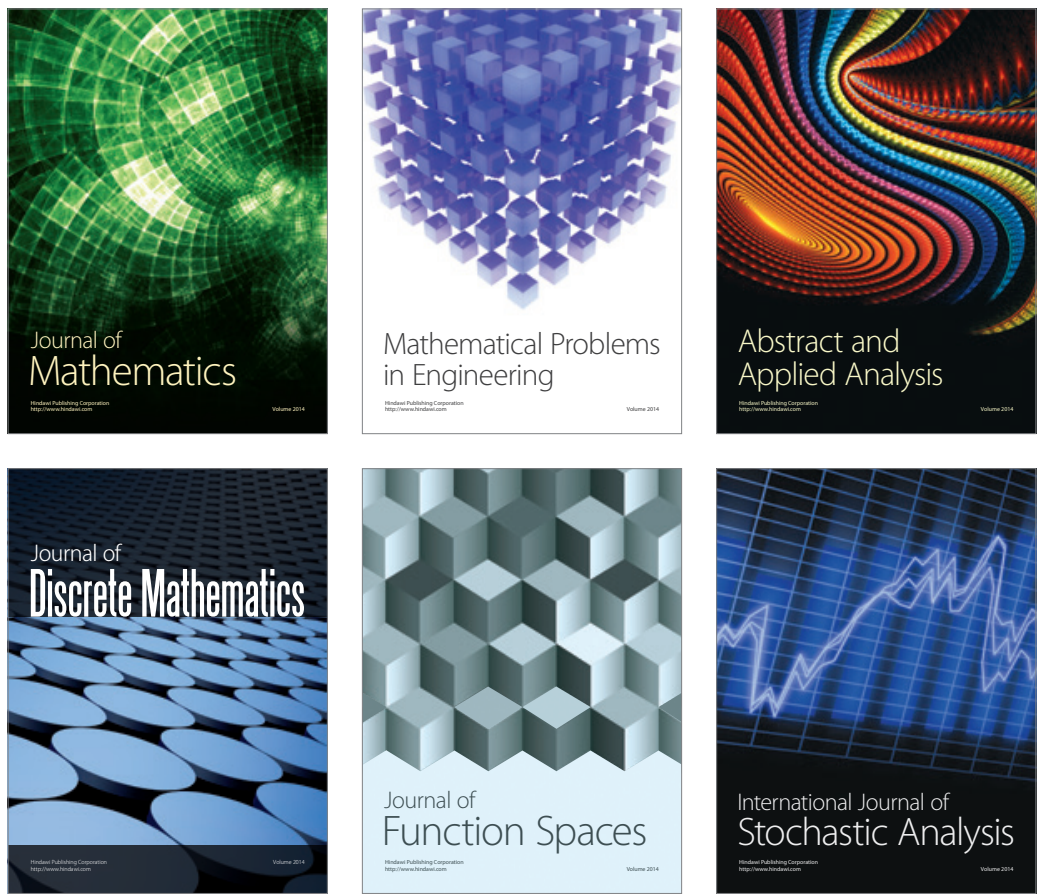

Journal of

Function Spaces

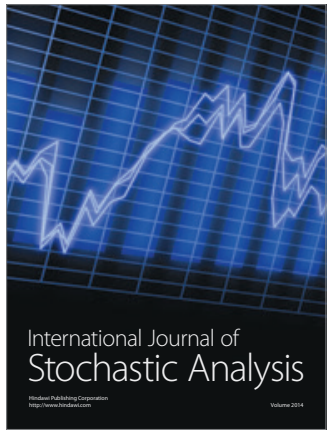

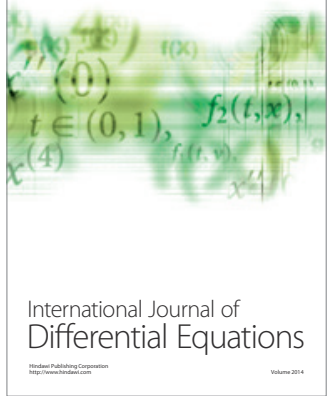
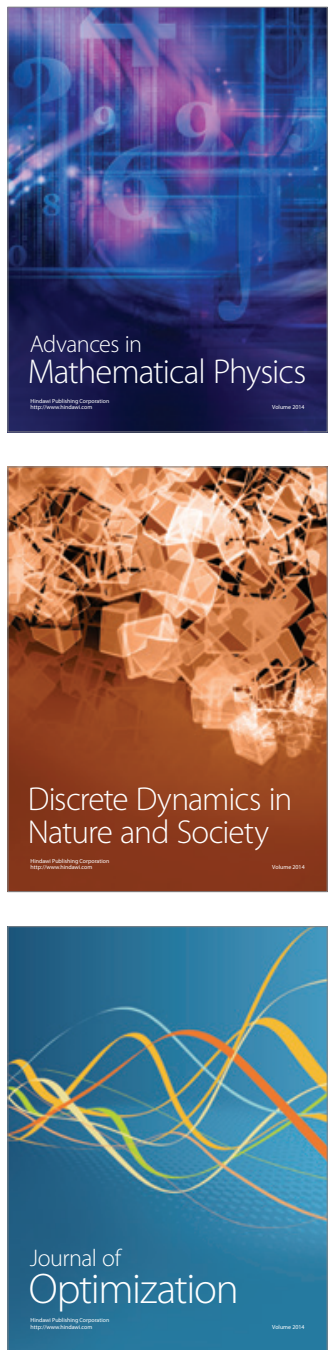